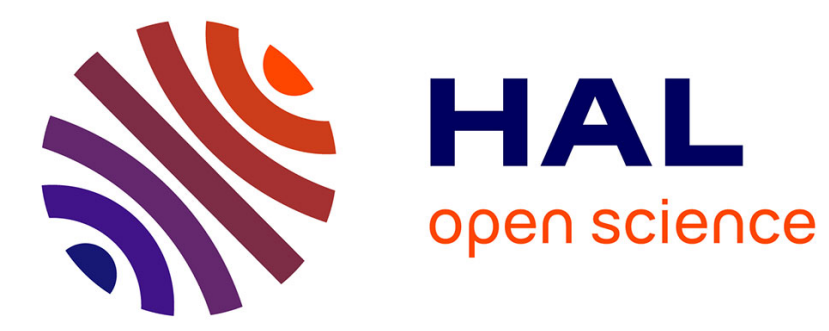

\title{
Compiling pattern matching to in-place modifications
}

Paul Iannetta, Laure Gonnord, Gabriel Radanne

\section{To cite this version:}

Paul Iannetta, Laure Gonnord, Gabriel Radanne. Compiling pattern matching to in-place modifications. GPCE 2021 - 20th International Conference on Generative Programming: Concepts \& Experiences, Oct 2021, Chicago \& Virtual, United States. 10.1145/3486609.3487204 . hal-03355377

\section{HAL Id: hal-03355377 \\ https://hal.science/hal-03355377}

Submitted on 18 Oct 2021

HAL is a multi-disciplinary open access archive for the deposit and dissemination of scientific research documents, whether they are published or not. The documents may come from teaching and research institutions in France or abroad, or from public or private research centers.
L'archive ouverte pluridisciplinaire HAL, est destinée au dépôt et à la diffusion de documents scientifiques de niveau recherche, publiés ou non, émanant des établissements d'enseignement et de recherche français ou étrangers, des laboratoires publics ou privés. 


\title{
Compiling pattern matching to in-place modifications ${ }^{*}$
}

\author{
(author long version)
}

\author{
Gabriel Radanne \\ Inria, EnsL, UCBL, CNRS, LIP \\ France \\ gabriel.radanne@inria.fr
}

\author{
Paul Iannetta \\ Univ Lyon, EnsL, UCBL, CNRS, Inria, \\ LIP \\ France \\ paul.iannetta@ens-lyon.fr
}

\author{
Laure Gonnord \\ University of Grenoble Alpes, \\ Grenoble INP, LCIS \& LIP \\ France \\ laure.gonnord@Icis.grenoble-inp.fr
}

\begin{abstract}
Algebraic data types and pattern matching are popular tools to build programs manipulating complex datastructures in a safe yet efficient manner. On top of its safety advantages, compilation techniques can turn pattern matching into highly efficient deconstruction code for immutable use cases.

Conversely, high-performance datastructures and languages prefer to leverage (controlled) mutations to maximize time and memory efficiency. Algebraic data types provide a natural framework to efficiently describe in-place transformations as rewrite rules. Such representation could take advantage of parallelism opportunities that appear in tree-like structures.

We present early steps towards a new technique to compile pattern matching as parallel in-place modifications of the underlying memory representation. Towards this goal, we combine the usual language approach which is common in pattern-matching compilation with tools from the polyhedral model, which is commonly used in high-performance code generation to output efficient $\mathrm{C}$ code. We present our formalism, along with a prototype implementation.
\end{abstract}

Keywords compilation, ADT, rewriting, code generation

\section{ACM Reference Format:}

Gabriel Radanne, Paul Iannetta, and Laure Gonnord. 2021. Compiling pattern matching to in-place modifications: (author long version). In Proceedings of the 20th ACM SIGPLAN InternationalConference on Generative Programming: Concepts and Experiences (GPCE'21), October 17-18, 2021, Chicago, IL, USA. ACM, New York, NY, USA, 9 pages. https://doi.org/10.1145/3486609.3487204

\section{Introduction}

Algebraic datatypes are used for a wide variety of purposes such as representing context (render trees, ray tracing, abstract syntax trees), or tree-based datastructures (AVL [1],

${ }^{*}$ This work was partially funded by the French National Agency of Research in the CODAS Project (ANR-17-CE23-0004-01)

Author Version - GPCE '21, October 17-18, 2021, (Virtual)

( 2021 Copyright held by the owner/author(s).

This is the author's version of the work. It is posted here for your personal use. Not for redistribution. The definitive Version of Record was published in Proceedings of the 20th ACM SIGPLAN InternationalConference on Generative Programming: Concepts and Experiences (GPCE'21), October 17-18, 2021, Chicago, IL, USA, https://doi.org/10.1145/3486609.3487204. red-black or B-Trees [10]). These structures are widely used in immutable functional languages, but also in high-performance contexts which generally rely on in-place mutations to modify the corresponding term.

Traditional techniques to optimize pattern matching [15] relies on immutability and don't leverage any parallelism. More recent work $[9,12,13,18,20]$ optimize terms used as container, notably by improving the parallelism and datalocality of traversals. However, these work severely limits the possibility of changing the structure of terms by only allowing mutations of values embedded in the structure.

The memory representation of terms is also a critical topic in obtaining good performances. This has been tackled both generally by providing optimized layouts for algebraic datatypes [20], and on particular cases through cache oblivious algorithm for high performance datastructures [4]. These representations are seldom used in functional programming due to their incompatibility with the high degree of sharing present in immutable datastructures [16]. However, they offer good data-locality which modern processors can take advantage of, notably through cache-oblivious and cacheaware algorithms, and lend themselves better to parallelism.

Many such representations exist (see. [5] for an overview). In the rest of this article, we assume that each subterm in a term is laid out in a set of layers addressable in constant time from the root of the subterm. Layers are considered indivisible and can be copied in a cache-friendly manner. This corresponds, for instance, to the Breadth-First Layout where elements of a term are laid out in an array in their breadth-first ordering. In this context, terms are a subpart of a support, which spans the whole underlying array.

Approach and contributions In this work, we attempt to optimize structural transformations on terms of algebraic data-types by exhibiting parallelism and pipelining. We describe a new approach to compile pattern matching as inplace modifications on flattened terms of algebraic datatypes. For this purpose, we define REw, a core language to rewrite algebraic terms (Section 2). We first use a languagebased approach to derive dependencies between operations on subterms (Section 3.1). We then rely on optimizing compilation techniques to schedule these operations (Section 3.2) and emit the appropriate sequential code (Section 4). We 
also developed a prototype implementation, which we use to illustrate our examples.

\section{From Pattern Matching to Memory Moves}

We now describe the Rew core language. Rew allows to describe Algebraic Data Types and simple rewriting rules on them. For conciseness, we omit the type system and dynamic semantic for this simple system (see $[11,15]$ for exhaustive descriptions of rich pattern languages) and focus on the compilation. We start with our running example before proceeding with the definition of the language.

Example 1 (Simple example of Rew program). As a running example, we consider the code below which defines the algebraic data type for binary trees containing integers and a transformation pull-up which pulls the right subtree up, also represented graphically. A REw function is similar to explicitly typed functions using pattern-matching, but defines a rewrite. Here, expressions only allow constructors and variables.
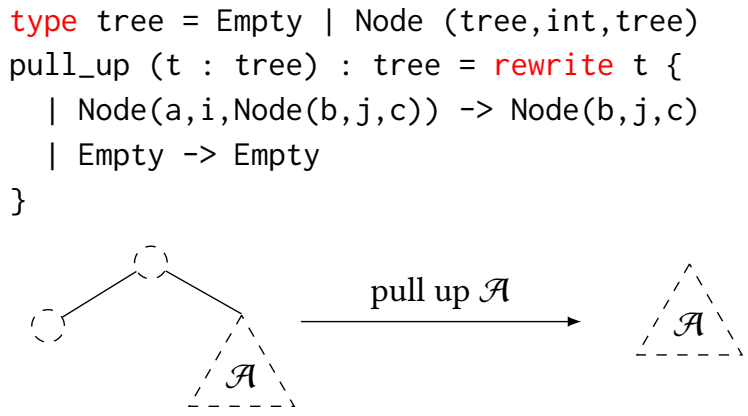

\subsection{The Rew language}

In the rest of this article, we use the following notations. We denote types $t$, expressions $e$ and patterns $p$. We use overbars for syntactic lists (for instance $\bar{p}$ is a list of patterns) and overarrows for vectors (for instance $\vec{k}$ ). The syntax of REw is given in Definition 1 and demonstrated in Example 1.

Types are either built-in or user-defined. We write Scalar the set of built-in scalar types such as integers, floatingpoints numbers, etc. For simplicity, we only consider nonparametric types, but our setting easily extends to parametric types, as long as the code is monomorphic (or specialized before-hand). A type declaration is composed of several constructors (written Constr $\in$ Constructors), each with several parameters. Expressions (resp. patterns) contain variables (resp. bindings) and constructors. A clause is a pair of a pattern and an expression. A program is a list of clauses.

Typing for such a language is a simple subset of typing in much richer languages [11]. As a single restriction, we consider that variables can never be re-defined. In the rest of this article, we assume the existence of an operator Type $(c, x)$ which gives the type of $x$ in the context of a clause $c$ (i.e., including bindings induced by the pattern part).
Definition 1 (Syntax of the rewrite language REw).

$$
\begin{aligned}
t & ::=\overline{\operatorname{Constr}\left(t_{0}, \ldots, t_{n}\right)} \mid t_{0} \in \text { Scalar } \\
p & ::=x \in \operatorname{Vars} \mid \operatorname{Constr}\left(p_{0}, \ldots, p_{n}\right) \\
e & ::=x \in \operatorname{Vars} \mid \operatorname{Constr}\left(e_{0}, \ldots, e_{n}\right) \\
c & ::=p \rightarrow e
\end{aligned}
$$

(Expressions)

(Clauses)

In the rest of this article, we compile each clause independently. As such, we now only consider a single clause $p \rightarrow e$, for which we will compute a set of elementary operations (copies) that should be performed. We propose an approach in two steps, described in the rest of the Section. We also derive a notion of dependance relation that captures a partial ordering for these operations (a read of a term should be performed before its use).

\subsection{Characterizing coarse grain memory moves}

The coarse-grain decomposition captures the structural memory moves to be performed: for instance, in the clause Node( $a, i, \operatorname{Node}(b, j, c)) \rightarrow \operatorname{Node}(b, j, c)$, we need to move the subterm corresponding to the variable $b$ to the first field of the constructor Node. For this purpose, Definition 2 presents the notions of location of a subterm in a term and of moves of a subterm from a location to another. A field is composed of an index and a type, written . $i / t$. A subterm location is a (potentially empty) list of fields, or an external location (for instance, an argument of the surrounding function). A subterm move is a variable binder annotated with its source and destination locations.

Definition 2 (Subterm locations and movements).

$$
\begin{aligned}
\mathrm{f}::=. i / t \quad i \in \mathbb{N} \\
\ell::=\overline{\mathrm{f}} \mid \text { External } \\
m::=\left(x: t \mid \ell \rightarrow \ell^{\prime}\right)
\end{aligned}
$$

We can compute the moves of a clause $p \rightarrow e$ through a traversal, as defined by the Moves function below. We assume the existence of the helper functions Vars, which gather all the variables of an expression or a pattern, and $\operatorname{Locs}(a, x)$ which obtain all the positions at which $x$ appears in the pattern or expression $a$. As additional restriction, a variable only appears once in a pattern (but potentially several times in an expression).

$$
\begin{aligned}
& \operatorname{Moves}(p \rightarrow e, t)= \\
& \left\{\begin{array}{l|l}
\left(x: t_{x} \mid \ell_{p} \rightarrow \ell_{e}\right) & \begin{array}{rl}
x & \in \operatorname{Vars}(p) \cup \operatorname{Vars}(e) \\
t_{x} & =\operatorname{Type}(p \rightarrow e, x)
\end{array} \\
\ell_{p}= \begin{cases}\operatorname{Locs}(p, x) & \text { if } x \in \operatorname{Vars}(p) \\
\emptyset & \text { otherwise }\end{cases} \\
\ell_{e} \in \begin{cases}\operatorname{Locs}(e, x) & \text { if } x \in \operatorname{Vars}(e) \\
\emptyset & \text { otherwise }\end{cases}
\end{array}\right\}
\end{aligned}
$$


Example 2. On the first clause in Example 1, we obtain the following moves:

$$
\begin{array}{cc}
\mid j: \text { int } \mid .2 / \text { tree. } 1 / \text { int } \rightarrow .1 / \text { int } ~ & \mid i: \text { int } \mid .1 / \text { int } \rightarrow \emptyset D \\
\mid b: \text { tree } \mid .2 / \text { tree. } 0 / \text { tree } \rightarrow .0 / \text { tree }) & \mid a: \text { tree } \mid .0 / \text { tree } \rightarrow \emptyset D \\
\mid c: \text { tree } \mid .2 / \text { tree. } 2 / \text { tree } \rightarrow .2 / \text { tree }) &
\end{array}
$$

Given a set of movements on subterms, we must decide if some movements should be done before the other. For instance, in Example 2, the moves of $b$ and $j$ must be executed before the move of $c$, as this last move will erase the location $.2 /$ tree, which originally contains $b$ and $j$. For this purpose, we define the notion of conflict between two locations.

Definition 3 (Conflict between locations). We say that $\ell$ and $\ell^{\prime}$ are in conflict, written $\ell \bowtie \ell^{\prime}$, if $\ell$ is prefix of $\ell^{\prime}$ or $\ell^{\prime}$ is prefix of $\ell$. External locations are never in conflict with anything. If $\ell \bowtie \ell^{\prime}$, we write $\operatorname{diff}\left(\ell, \ell^{\prime}\right)$ the extra suffix.

We can give a primitive notion of "must happen before" relation on moves: Given two moves $\left(a: t \mid \ell_{p} \rightarrow \ell_{e}\right)$ and $\left(a^{\prime}: t^{\prime} \mid \ell_{p}^{\prime} \rightarrow \ell_{e}^{\prime}\right), a$ must happen before $a^{\prime}$ if $\ell_{p} \bowtie \ell_{e}^{\prime}$.

\subsection{Fine grain decomposition into memory moves}

The moves we have shown so far operates on subterms, i.e. a given location and all its descendants. This coarse-grained approach causes two issues. First, it induces more conflicts than necessary, making the "happens before" less precise. Indeed, any subterm will trigger a conflict, even if other part of the term could be modified independently. Second, it means moves will easily conflict with themselves, as is the case of the move of $c$ in Example 2. In particular, it is not clear at this stage how we could implement the move of $c$.

To alleviate these problems, we leverage the memory representation mentioned in Section 1 by decomposing each subtree move into a collection of memory moves on paths. Definition 4 gives the notion of path $\pi$ which extends locations with repetitions indexed by an iteration variable $k$. Path also include wildcards $\varphi$ which correspond to any field. These wildcards allow separating the representation by layers: $\varphi^{k}$ is the $k^{\text {th }}$ layer of a subterm. Memory moves are moves operating on memory paths. Paths correspond to regular expressions on locations without alternatives and of star height 1 . Matching is immediate by treating named repetitions as Kleene stars.

Definition 4 (Paths and memory movements).

$$
\begin{array}{rrr}
k & \in \mathrm{ItVars} & \text { (Iteration variables) } \\
\pi::=\ell \cdot \pi\left|\ell^{k} \cdot \pi\right| \varphi^{k} \mid \varepsilon & \text { (Path) } \\
m_{\pi}::=\left(\pi \rightarrow \pi^{\prime}\right) & \text { (Memory Move) }
\end{array}
$$

In the rest, we freely use properties of regular languages on paths.
The Atomize function aims to decompose subtree movements (where the iteration is implicit) into memory movements with explicit iteration. On the way, it eliminates spurious "self-conflict", i.e., rules whose source and destinations are in conflict and reveal potential parallelism for later phases. We first look at the output of Atomize on an example.

Example 3. In Example 2, we obtained the followind subterm moves for the first clause of Example 1:

$$
\begin{aligned}
& \emptyset j: \text { int } \mid .2 / \text { tree. } 1 / \text { int } \rightarrow .1 / \text { int }) \quad(i: \text { int } \mid .1 / \text { int } \rightarrow \emptyset) \\
& \mid b: \text { tree } \mid .2 / \text { tree. } 0 / \text { tree } \rightarrow .0 / \text { tree }) \quad(a: \text { tree } \mid .0 / \text { tree } \rightarrow \emptyset D \\
& \text { (c: tree } \mid .2 / \text { tree. } 2 / \text { tree } \rightarrow .2 / \text { tree })
\end{aligned}
$$

The tree can be further decomposed into independant layers (eg. in the case of the breadth first search layout, layers are regions of the form $\left.\left(2^{i}, 2^{i+1}\right)\right)$, the memory moves can thus be further broken down into generalized moves which are moves about memory regions. In the end of the application of Atomize we will find the following moves, where we sometimes shorten paths of the form $f \cdot f^{k}$ as $f^{k+1}$.

$$
\begin{aligned}
0.0 / \text { tree. } \varphi^{k_{0}} & \rightarrow \text { External } \\
0.1 / \text { int }^{2} & \rightarrow \text { External }) \\
\left(.2 / \text { tree. } 0 / \text { tree. } \varphi^{k_{1}}\right. & \rightarrow .0 / \text { tree. } \varphi^{k_{1}} \\
(.2 / \text { tree. } 1 / \text { int } & \rightarrow .1 / \text { int }) \\
\left((.2 / \text { tree })^{k_{2}+2}\right. & \rightarrow(.2 / \text { tree })^{k_{2}+1} D \\
\left((.2 / \text { tree })^{k_{2}+2} .0 / \text { tree. } \varphi^{k_{3}}\right. & \left.\rightarrow(.2 / \text { tree })^{k_{2}+1} \cdot 0 / \text { tree. } \varphi^{k_{3}}\right) \\
\left((.2 / \text { tree })^{k_{2}+2} \cdot 1 /\right. \text { int } & \left.\rightarrow(.2 / \text { tree })^{k_{2}+1} .1 / \text { int }\right)
\end{aligned}
$$

The rules $(i)$ and $(j)$ correspond directly to the subterm moves: Since those terms are scalar, they do not require any iteration. ( $a$ ) and (b) correspond to moves on $a$ and $b$. Since source and destination do not conflict, we simply copy each layer separately. $\ell . \varphi^{k}$ here denotes the $k^{\text {th }}$ layers of the subterm anchored in $\ell$ and is used to copy a subterm layers by layers. The subterm move for (c), on the other hand, has conflicting source and destination and requires additional care. We decompose it into several memory moves, corresponding to climbing the "stair" of subterms along the direction.2/tree. This is schematized on Fig. 1, which represents the memory layout of a term of type tree, along with the new memory movements in bold arrows and the iteration directions in double arrows. The memory movement $(c)$ is on the stair itself, while (c1) and (c0) correspond to all the potential subterms which are not reached by the prime iteration direction.2/tree. Depending of whether such subterms are scalars or terms, we decompose them further into layers.

Let us now define auxiliary functions used in Atomize.

Definition 5 (Type of a location). The type of a non-external location is Type $(\ell \cdot x / T)=T$.

Definition 6 (Complement of a location). Let a location $\ell$ and a type $t=\overline{\operatorname{Constr}\left(\overline{t_{i}}\right)}$. We consider $\mathcal{F}$ the sets of all the fields $\left(. i / t_{i}\right)$ present in $t$. The complement of $\ell$ in $t$, written $\operatorname{Compl}(t, \ell)$, is the set of paths in $t$ which are not $\ell$. It allows 


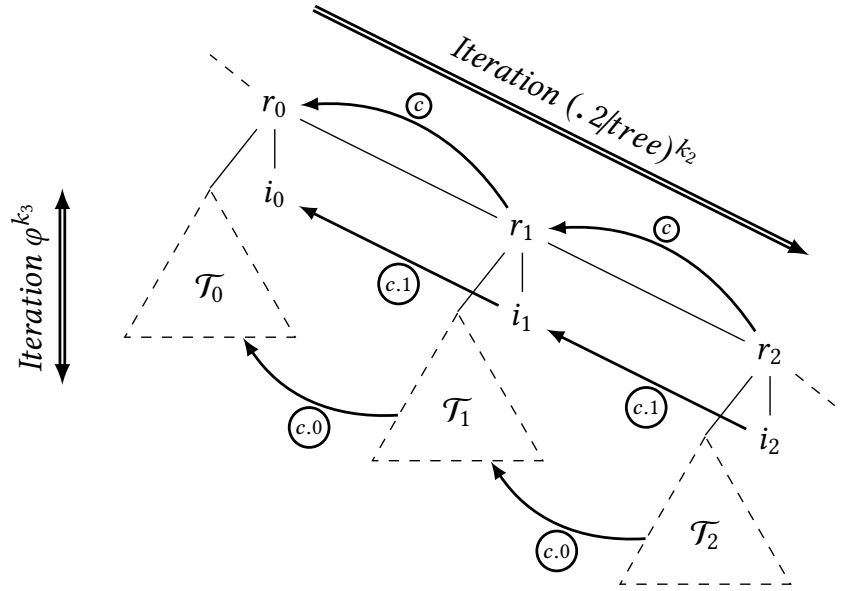

Figure 1. Memory movements $(c),(c 0),(c 1)$ in Example 1.

us to inspect all subterms which are not in the prime iteration direction. We define:

$$
\begin{aligned}
\operatorname{Compl}(t,[]) & =\{\} \\
\operatorname{Compl}\left(t, . i / t^{\prime} . \ell\right) & =\left(\mathcal{F}-. i / t^{\prime}\right) \cup\left\{. i / t^{\prime} . \ell^{\prime} \mid \ell^{\prime} \in \operatorname{Compl}\left(t^{\prime}, \ell\right)\right\}
\end{aligned}
$$

The complete definition of Atomize is shown in Algorithm 1. The first two cases are simple: identity moves are removed, and scalar moves are kept as-is, as they are nonrecursive. The treatment of subterm moves depend if they have a self-conflict. If they do not, we move each layer, which is schematized by the move $\left(\ell_{p} \cdot \varphi^{k} \rightarrow \ell_{e} \cdot \varphi^{k}\right)$. Note that all such moves are disjoint (neither sources nor destinations can overlap). This hints at the possibility of parallelizing such loop later on. In case of self-conflict, we decompose this move further by choosing an iteration direction. The conflict gives us a natural choice: since one location is the prefix of the other, we use the extra suffix $\ell=\operatorname{diff}\left(\ell_{p}, \ell_{e}\right)$ to direct the iteration. All the subterms not present along the iteration direction are then given by $\operatorname{Compl}(\ell)$. We inspect these subterms and create new appropriate moves depending on their types. By construction of Compl, none of these moves overlap (with themselves nor with each other).

Every single move has a domain, which is the set of valid values that can be taken by its iteration variables, and computed as a function parametrized by a formal parameter $N$, that denotes an upper bound on the height of terms embedded in the underlying array support. The domain of a move is directly induced by the admissible lengths of its paths.

Definition 7 (Length of a path). Given a path $\pi$, its admissible length, written $|\pi|$, is the length of any location that could match the given path. It is the linear form defined as:

$$
\begin{aligned}
|\ell . \pi| & =|\ell|+|\pi| & \left|\varphi^{k}\right| & =k \\
\left|\ell^{k} . \pi\right| & =|\ell| * k+|\pi| & |\varepsilon| & =0
\end{aligned}
$$

Since this is a linear form on $\vec{k}$, we write $L_{\pi}$ and $\vec{l}_{\pi}$ such that $|\pi|(\vec{k})=L_{\pi} \cdot \vec{k}+\vec{l}_{\pi}$
Definition 8 (Domain of a move). We consider a move $m=\left(\pi \rightarrow \pi^{\prime}\right)$. The domain of $m$ is written $\mathcal{D}_{m}$ and defined

$\mathcal{D}_{m}=\left\{\vec{k} \mid(0 \leq|\pi|(\vec{k}) \leq N) \wedge\left(0 \leq\left|\pi^{\prime}\right|(\vec{k}) \leq N\right) \wedge(\overrightarrow{0} \leq \vec{k})\right\}$

Since $|\pi|$ is a linear form on $\vec{k}$, the domain can also be defined as a polyhedron. We write $D_{m}$ and $\vec{d}_{m}$ such that $\mathcal{D}_{m}=\left\{\vec{k} \mid D_{m} \vec{k}+\vec{d}_{m} \geq \overrightarrow{0}\right\}$

\section{Memory moves scheduling}

Now that we have a fine grain characterisation of memory moves, we need to schedule them in order to generate code. We propose a two-steps approach, classical in the HPC community: first, we compute a compact representation for (read/write) dependencies, and from this representation, use optimisation to compute logical dates compatible with these dependencies with the help of an optimisation algorithm.

\subsection{Dependencies computation}

A dependency happens if two moves might potentially overlap. Since the language of expression in REw is pure, the only dependencies are induced directly by the rewriting specification: the left hand side acts as "reads" and the right hand side as "writes" on the specified paths. Usually, there are two types of conflicts: read-write, and write-write. However, in our case, a write-write conflict would mean two moves have the same destination. Since locations are entirely determined by positions in the AST of the right hand side, this is syntactically impossible.

Therefore, we only consider read-write dependencies, i.e., between the source of a move and the destination of another move. This naturally give rises to an ordered "happensbefore" relation on moves since the write should be done after the read. Furthermore, not only do we want a relation

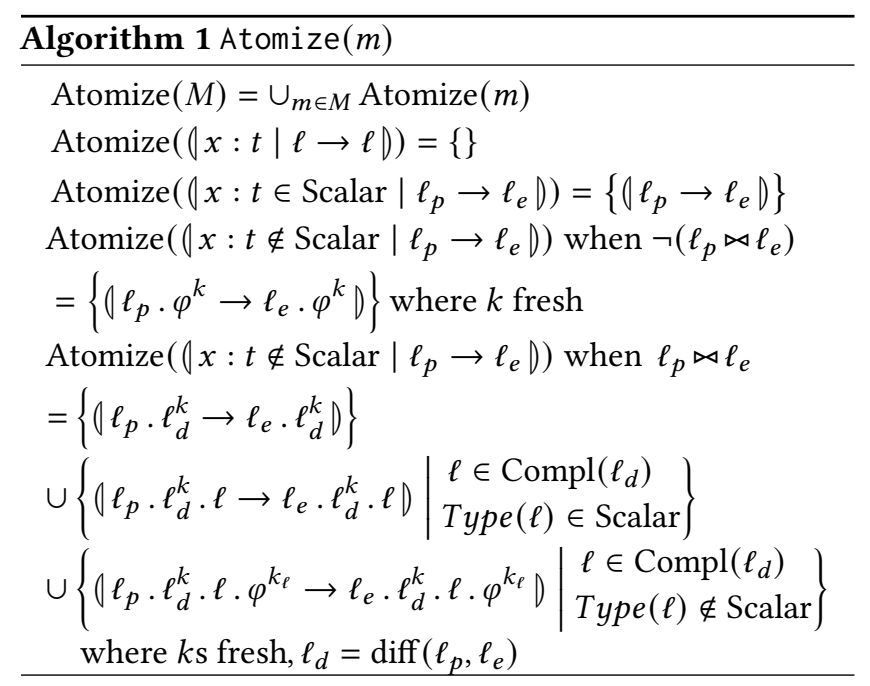


to indicate potential dependencies, but also when this dependency happens, in term of the iteration variables $k$. We thus annotate this relation with constraints on the $k \mathrm{~s}$.

Definition $9((\mathrm{R} / \mathrm{W})$ Dependencies between moves). We consider two moves $m=\left(\pi_{p} \rightarrow \pi_{e}\right)$ and $m^{\prime}=\left(\pi_{p}^{\prime} \rightarrow\right.$ $\pi_{e}^{\prime} \downarrow$ The dependencies between (the source of) $m$ and (the destination of ) $m^{\prime}$ is written $Q_{\left(m, m^{\prime}\right)}$ and defined as all values of the source and destination iteration vectors for which the memory paths actually intersect (do not have an empty intersection). Given $\mathcal{L}(\pi)$ the set of locations of $\pi$, we have:

$Q_{\left(\mid \pi_{p} \rightarrow \pi_{e} \emptyset,\left(\pi_{p}^{\prime} \rightarrow \pi_{e}^{\prime} \emptyset\right)\right.}=\left\{\left(\begin{array}{c}\vec{k} \\ \overrightarrow{k^{\prime}}\end{array}\right) \mid \exists \ell \in \mathcal{L}\left(\pi_{p}(\vec{k})\right) \cap \mathcal{L}\left(\pi_{e}^{\prime}\left(\vec{k}^{\prime}\right)\right)\right\}$

Computing $Q_{\left(m, m^{\prime}\right)}$ as a finite description such as a polyhedron is not immediate. Paths are not "usual" regular expressions since the $k s$ are symbolic, not concrete integers. In practice, we can obtain an exact representation of $Q_{\left(m, m^{\prime}\right)}$ thanks to careful syntactic manipulations on paths.

Lemma 1. $Q_{\left(m, m^{\prime}\right)}$ is a union of polyhedrons and computing its finite representation is decidable.

Example 4. (Domain and Dependencies) We now give the domain and dependencies of some memory moves from Example 3 We recall the moves $(b)$ and $(c 0)$.

$$
\begin{aligned}
& \text { (.2/tree. } \left.0 / \text { tree. } \varphi^{k_{1}} \rightarrow .0 / \text { tree. } \varphi^{k_{1}}\right) \\
& \left.(\text { (.2/tree })^{k_{2}+2} .0 / \text { tree. } \varphi^{k_{3}} \rightarrow(.2 / \text { tree })^{k_{2}+1} .0 / \text { tree. } \varphi^{k_{3}}\right)
\end{aligned}
$$

The domains are computed from the length of the paths. Given the formal parameter $N$, we have:

$$
\begin{aligned}
\mathcal{D}_{b} & =\left\{k_{1} \mid 0 \leq k_{1} \wedge k_{1}+2 \leq N\right\} \\
\mathcal{D}_{c 0} & =\left\{\left(k_{2} k_{3}\right) \mid 0 \leq k_{2} \wedge 0 \leq k_{3} \wedge k_{2}+k_{3}+3 \leq N\right\}
\end{aligned}
$$

We also compute the following dependencies:

$$
\begin{array}{rlr}
Q_{b, c 0} & =\left\{\left(k_{1} k_{2}^{\prime} k_{3}^{\prime}\right) \mid k_{2}^{\prime}=0 \wedge k_{3}^{\prime}=k_{1}\right\} & Q_{b, b}=\emptyset \\
Q_{c 0, c 0} & =\left\{\left(k_{2} k_{3} k_{2}^{\prime} k_{3}^{\prime}\right) \mid k_{2}+1=k_{2}^{\prime} \wedge k_{3}=k_{3}^{\prime}\right\} &
\end{array}
$$

We remark that the movement ( $b$ ) must be done before $(c 0)$, as they both access the memory path.2/tree.0/tree and all its descendant. We also remark that ( $c 0)$ has a self dependency, which induces an order of iteration along its first direction $k_{2}$. The direction $k_{3}$ doesn't have such an imposed order, which hints at later parallelism opportunities.

\subsection{Scheduling via constraint solving}

At the end of the previous section, for each clause of our Rew program, we obtained a tuple $(\mathcal{M}, \mathcal{T})$ where:

- each move $m \in \mathcal{M}$ has an application domain $\mathcal{D}_{m}$.

- each pair of moves carries a "dependency constraint" $Q_{m, m^{\prime}}$. Our objective is now to compute a valid schedule for the set of moves of the initial clause, i.e., an order for the subcomputations. We adapt in this section an approach based on constraint solving used in polyhedral compilation [7, 8, 14] as well as in termination proofs $[2,6]$.
A schedule is a function that assigns positive logical dates to each move computation such that all dependencies are satisfied (a computation that depends on another one is done strictly after). This is captured in Definition 10.

Definition 10. Schedule constraints: A schedule for the graph $(\mathcal{M}, \mathcal{T})$ is a function $\rho: \mathcal{M} \times \mathbb{Z}^{n} \rightarrow \mathbb{N}^{d}$, from the graph vertices to $\mathbb{N}^{d}$, which is positive:

$$
\vec{k} \in \mathcal{D}_{m} \Rightarrow \rho(m, \vec{k}) \geq \overrightarrow{0} \text { (component-wise) }
$$

(Positivity)

and whose values stricly increase (according to $\varsigma_{d}$, the standard lexicographic order on integer vectors) at each edge $t=\left(m, m^{\prime}\right) \in \mathcal{T}:$

$$
Q_{m, m^{\prime}}\left(k, k^{\prime}\right) \Rightarrow \rho(m, \vec{k}) \prec_{d} \rho\left(m^{\prime}, \vec{k}^{\prime}\right)
$$

(Increasing)

It is said affine if it is affine in the second parameter (the variables $\vec{k}$ ). If $d>0$ the schedule is said to be multi-dimensional of dimension $d$.

Remark. Schedules can be parallel, indeed there is no constraint forcing two non conflicting moves to happen one before the other. The computation of a valid schedule might thus find equal dates for two different moves.

Searching for one dimensional schedules First, we relax the increasing constraint (Increasing), for $d=1$, into:

$$
\left(\vec{k}, \vec{k}^{\prime}\right) \in Q_{t} \Rightarrow 0 \leq \rho\left(m^{\prime}, \vec{k}^{\prime}\right)-\rho(m, \vec{k}) \leq \epsilon_{t} \leq 1
$$

We now look for affine schedules, that is $\vec{c}, c_{0}$ such that $\rho(m, \vec{k})=\vec{c} \cdot \vec{k}+c_{0}$. Unfortunately, inlining this form leads to quadratic constraints $\vec{k} \in \mathcal{D}_{m} \Rightarrow \overrightarrow{\mathbf{c}} \cdot \overrightarrow{\mathbf{k}}+c_{0} \geq \overrightarrow{0}$. However, we can linearize these constraints using the Farkas lemma [19] (since $\mathcal{D}_{m}$ and $Q_{m, m^{\prime}}$ are polyhedra). This construction is detailed in Appendix A (author version only)

Lemma 2 (Constraints $C$ ). There exists a computable affine set of constraints $C$ computed from $(\mathcal{M}, \mathcal{T})$ that exactly describes the set of admissible schedules.

Finding a valid schedule consists in solving this set of constraints with an appropriate objective function (Algorithm 2). If a valid schedule exists, all $\epsilon_{t}$ are equal to 1 . Otherwise, we have a partial schedule, that we will complete in the next section.

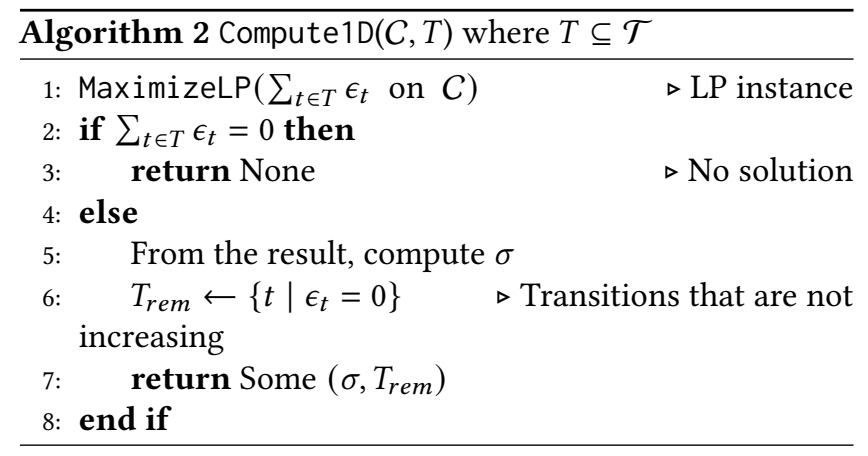




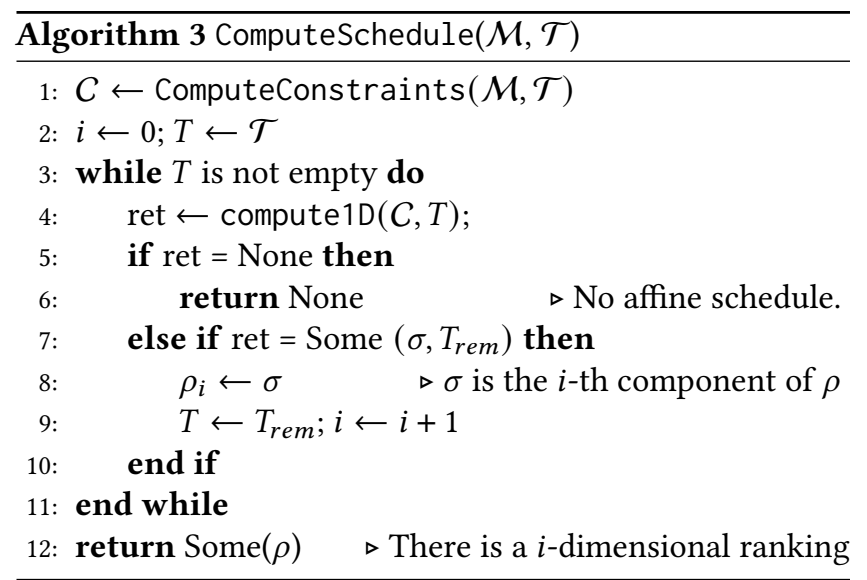

Multidimensional schedules As all schedules are not of dimension 1, we use a greedy algorithm, described in Algorithm 3 , similar to $[6,8,14]$ where each component of the schedule $\rho$ is constructed one after the other. At each loop it makes a call to compute $1 \mathrm{D}(C, T)$. If it succeeds, the number of constraints still to be satisfied have strictly decreased and we can relaunch the procedure on the system without these constraints (Line 9). Otherwise, the procedure ends without concluding. Surprisingly, despite this greedy approach, this technique is proven complete (if the dependencies are exact [2]), thus it always gives an affine schedule.

Example 5. (Schedule) From Example 4 we obtain the following schedules for $(b),(c 0),(c 1)$ :

$$
\rho(b)=\left(0, k_{1}\right) \quad \rho(c 0)=\left(k_{2}+1, k_{3}\right) \quad \rho(c 1)=\left(k_{2}+1,0\right)
$$

As $k_{2} \geq 0$, this schedule successfully captures that movement (b) must be done before (c0). Similarly, each $c 0\left(k, k^{\prime}\right)$ is done before before $c 0\left(k+1, k^{\prime}\right)$, as expected.

\section{Code emission}

The previous section provides us with a schedule $\rho(m, \vec{k})$ for each move of a given clause, the objective is now to generate a sequence of loop nests that will compute each (set of) moves in the order specified by the schedule, without forgetting any subcomputation. We use an algorithm inspired by previous works on code generation for the polyhedral framework [3, 17] depicted in Algorithm 4. It contains an inner procedure LOOPGEN which iterates recursively over the polyhedra to create a tree of nested loops.

At recursive call $i$, LOOPGEN generates the sequence of loops corresponding to dimension $i$ of the schedule $\rho$. At this point, we collect the projection of the polyhedra along dimension $i$ which we partition and merge with the procedure MergePolyhedra. This gives us a list of polyhedra which delimit the inner loops strictly inside $i$, on which we recursively call LoopGen. When $i=d$, we emit the moves contained in the sub-polyhedra obtained by the recursive

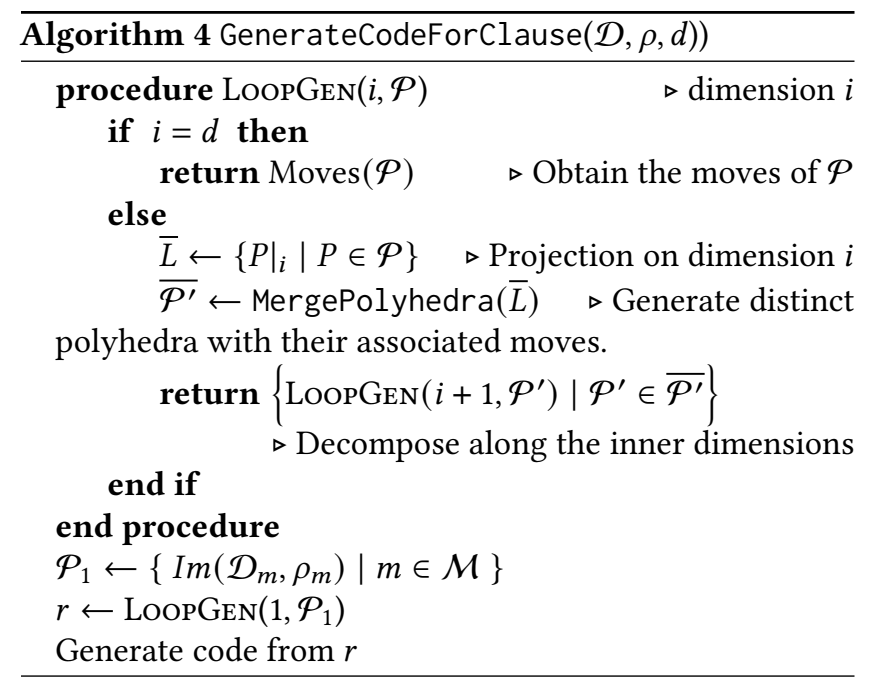

partitions. We initialize the set of polyhedrons with the set of images of $\mathcal{D}_{m}$ by $\rho_{m}$.

Example 6. If we recall Example 3, we can see that there are 5 moves. The first two are telling us that the subtree a is going into the void and should be ignored, and the last which is about coping over the structure will also be ignored. It remains to generate code for the three last ones. On the running example, we denote by $(i, j)$ the iteration dimensions. The initial set $\mathcal{P}_{1}$ contains the three following images:

$$
\begin{aligned}
\operatorname{Im}\left(\rho_{b}, \mathcal{D}_{b}\right) & =\{i=0 \text { and } 0 \leq j \leq N-2\} \\
\operatorname{Im}\left(\rho_{c 1}, \mathcal{D}_{c 1}\right) & =\{1 \leq i \leq N-2 \text { and } j=0\} \\
\operatorname{Im}\left(\rho_{c 0}, \mathcal{D}_{c 0}\right) & =\{1 \leq i \leq N-2 \text { and } 0 \leq j \leq N-i-2\} .
\end{aligned}
$$

From these polyhedra, we can build a partition on the first dimension: first, projecting the former images on the first dimension $i$ gives three polyhedra $P_{1}=\{i=0\}$ (associated to $b$ ) and $\left.P_{2}\right) P_{3}=\{1 \leq i \leq N-2\}$ (associated with $c 0, c 1$ moves), and then The partition is then $\overline{\mathcal{P}^{\prime}}{ }_{1}=\left[\left(P_{1}, b\right),\left(P_{2},\{c 0, c 1\}\right)\right]$ (we track the associated moves). The polyhedra $P_{1}$ and $P_{2}$ encode the outermost iterations necessary to compute memory moves for $b$ and $c 0, c 1$. In the final code these polyhedra will generate two successive for loops. It now remains to generate their inner body.

The two other recursive calls to LOOPGEN(g)enerate inner loops inside these " $P_{1}, P_{2}$ loops". Let us focus on the $(c 0, c 1)$ part. From projections on $j$ we obtain two polyhedra $P_{1,2}^{\prime}=\{j=0\}$ and $P_{2,2}^{\prime}=\{0 \leq j \leq N-i-2\}$ that depict the iterations for $c 1$ and c0 respectively, it remains to generate the corresponding for loops and their body. The final code is thus ${ }^{1}$ :

\footnotetext{
${ }^{1}$ Each move is implemented as a memcopy of adjacent cells.
} 


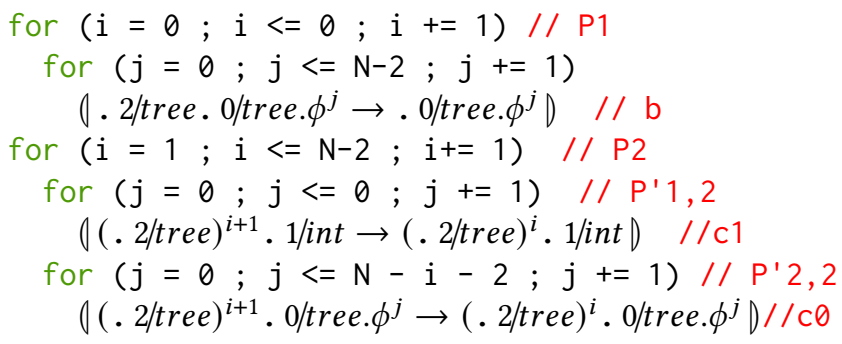

\section{Future work and conclusion}

We have presented the early work towards a framework to optimize in-place pattern matching on algebraic data types. So far, the framework we have presented is limited. We plan to extend it via a number of new features which are essential to improve its applicability.

\subsection{An end-to-end implementation}

We have implemented the first two steps (Sections 2 and 3) as a prototype ${ }^{2}$. The last step is so far done using standard tooling from the HPC community which computes loop nests based on a schedule. We aim to complete and extend this implementation with the features mentioned next.

\subsection{Richer expression language}

A richer expression language is essential to extend the scope of this work. We can in particular sketch the following extensions.

Functions on scalars Our expression language can be easily extended to arbitrary functions on scalars such as arithmetic operations. In practice, moves become instructions of the form $\pi \leftarrow f\left(\pi_{0}, \ldots, \pi_{n}\right)$. The notion of domain and dependencies immediately extend to this new context.

Functions on terms Our framework transforms a function on terms into a set of memory movements. This leads to a notion of "inlining" for function calls on subterms, since we know the prefix for both source and destinations of the nested rules. Once inlined, the rules behaved as currently, and all rules can be scheduled and compiled together.

Self-recursion The previous remark on inlining gives a way to handle some limited form of self-recursion. Indeed, we can inline the function itself at the position of the recursive call. More concretely, given the type of tree in Example 1 and a clause of the form $\operatorname{Node}(a, i, b) \rightarrow \operatorname{Node}(f a, i, f$ b), it suffices to prefix all the moves by $(.0 / \text { tree } \mid .2 / \text { tree })^{k}$. This prefix spans all the subterms on which $f$ is called recursively. This requires adding alternatives inside paths. We believe our techniques to compute domains and dependencies still hold up with this extension. While this is not a fully general recursion scheme, it applies to functions such as map or transformations such as constant folding.

\subsection{Richer pattern language: guards}

Guards, i.e., Boolean tests inside patterns, can easily be added to our framework by equipping moves with conditions governing their applicability. This would have the added benefit to allow us to compile the whole pattern matching, including the choice of which pattern to apply. The difficulty here lies in emitting code which properly factorize the tests during iterations to avoid breaking memory locality.

\subsection{Better code generation}

Parallel code generation In this article, we only present sequential, fairly naive, code generation. We have only scratched the surface of what the polyhedral model can offer in terms of highly optimized code generation. In particular, our setup is ideally designed to produce parallel and vectorized code. We can also leverage our knowledge of the memory layout to improve memory locality when iterating through subterms.

Improve code size Currently, we do not present any technique to improve the size of the generated code unlike the papers $[3,17]$ we use as our basis, which are remove dead code if any and try to unify single point polyhedra with adjacent polyhedra.

\footnotetext{
${ }^{2}$ https://github.com/Drup/adtr/
} 


\section{References}

[1] G. M. Adel'son-Vel'skii and E. M. Landis. 1962. An algorithm for organization of information. Dokladi Akademia Nauk SSSR 146, 2 (April 1962), 263-266.

[2] Christophe Alias, Alain Darte, Paul Feautrier, and Laure Gonnord. 2010. Multi-dimensional Rankings, Program Termination, and Complexity Bounds of Flowchart Programs. In Static Analysis Symposium. Perpignan, France. https://doi.org/10.1007/978-3-642-15769-1

[3] C. Bastoul. 2004. Code generation in the polyhedral model is easier than you think. In Proceedings. 13th International Conference on Parallel Architecture and Compilation Techniques, 2004. PACT 2004. 7-16. https: //doi.org/10.1109/PACT.2004.1342537

[4] Michael A. Bender, Jeremy T. Fineman, Seth Gilbert, and Bradley C. Kuszmaul. 2005. Concurrent Cache-Oblivious b-Trees. In Proceedings of the Seventeenth Annual ACM Symposium on Parallelism in Algorithms and Architectures (SPAA '05). Association for Computing Machinery, New York, NY, USA, 228-237. https://doi.org/10.1145/1073970.1074009

[5] Gerth Stølting Brodal, Rolf Fagerberg, and Riko Jacob. 2002. Cache Oblivious Search Trees via Binary Trees of Small Height. In Proceedings of the Thirteenth Annual ACM-SIAM Symposium on Discrete Algorithms (SODA'02). Society for Industrial and Applied Mathematics, USA, 3948.

[6] Michael A. Colón and Henny B. Sipma. 2002. Practical Methods for Proving Program Termination. In 14th International Conference on Computer Aided Verification (CAV) (Lecture Notes in Computer Science), Vol. 2404. Springer Verlag, 442-454.

[7] Paul Feautrier. 1992. Some Efficient Solutions to the Affine Scheduling Problem, I, One-dimensional Time. International fournal of Parallel Programming 21, 5 (October 1992), 313-348.

[8] Paul Feautrier. 1992. Some Efficient Solutions to the Affine Scheduling Problem, II, Multi-dimensional Time. International fournal of Parallel Programming 21, 6 (December 1992), 389-420.

[9] Michael Goldfarb, Youngjoon Jo, and Milind Kulkarni. 2013. General Transformations for GPU Execution of Tree Traversals. In Proceedings of the International Conference on High Performance Computing, Networking, Storage and Analysis (SC '13). Association for Computing Machinery, New York, NY, USA, Article 10, 12 pages. https://doi.org/10.1145/2503210.2503223

[10] Gaston H. Gonnet and Ricardo Baeza-Yates. 1991. Handbook of Algorithms and Data Structures in Pascal and C (2nd ed.). Addison-Wesley Pub (Sd).

[11] Sebastian Graf, Simon Peyton Jones, and Ryan G. Scott. 2020. Lower your guards: a compositional pattern-match coverage checker. Proc. ACM Program. Lang. 4, ICFP (2020), 107:1-107:30. https://doi.org/10. $1145 / 3408989$

[12] Youngjoon Jo and Milind Kulkarni. 2011. Enhancing Locality for Recursive Traversals of Recursive Structures. In Proceedings of the 2011 ACM International Conference on Object Oriented Programming Systems Languages and Applications (OOPSLA '11). Association for Computing Machinery, New York, NY, USA, 463-482. https://doi.org/10.1145/ 2048066.2048104

[13] Youngjoon Jo and Milind Kulkarni. 2012. Automatically Enhancing Locality for Tree Traversals with Traversal Splicing. In Proceddings of the ACM International Conference on Object Oriented Programming Systems Languages and Applications (OOPSLA '12). Association for Computing Machinery, New York, NY, USA, 355-374. https://doi.org/ 10.1145/2384616.2384643

[14] R. M. Karp, R. E. Miller, and S. Winograd. 1967. The Organization of Computations for Uniform Recurrence Equations. 7. ACM 14, 3 (July 1967), 563-590.

[15] Luc Maranget. 2008. Compiling pattern matching to good decision trees. In Proceedings of the ACM Workshop on ML, 2008, Victoria, BC, Canada, September 21, 2008, Eijiro Sumii (Ed.). ACM, 35-46. https: //doi.org/10.1145/1411304.1411311
[16] Chris Okasaki. 1999. Purely functional data structures. Cambridge University Press.

[17] Fabien Quilleré, Sanjay Rajopadhye, and Doran Wilde. 2000. Generation of Efficient Nested Loops from Polyhedra. International fournal of Parallel Programming 28 (10 2000), 469-498. https://doi.org/10.1023/A: 1007554627716

[18] Laith Sakka, Kirshanthan Sundararajah, Ryan R. Newton, and Milind Kulkarni. 2019. Sound, Fine-Grained Traversal Fusion for Heterogeneous Trees - Extended Version. CoRR abs/1904.07061 (2019). arXiv:1904.07061 http://arxiv.org/abs/1904.07061

[19] A. Schrijver. 1986. Theory of linear and integer programming. Wiley, NewYork.

[20] Kirshanthan Sundararajah and Milind Kulkarni. 2019. Composable, Sound Transformations of Nested Recursion and Loops. In Proceedings of the 40th ACM SIGPLAN Conference on Programming Language Design and Implementation (PLDI 2019). Association for Computing Machinery, New York, NY, USA, 902-917. https://doi.org/10.1145/ 3314221.3314592 


\section{A Detailed construction of the linear constraint system of Lemma 2}

In this section we explain how to obtain the constraint system $C$ to solve to obtain a valid schedule of our program (Lemma 2). The final algorithm is depicted in Algorithm 5.

We recall that a schedule is a function that assigns positive logical dates to each move computation such that all dependencies are satisfied (a computation that depends on another one is done strictly after). This is captured in Definition 10 that we recall here:

Definition 11. Schedule constraints: A schedule for the graph $(\mathcal{M}, \mathcal{T})$ is a function $\rho: \mathcal{M} \times \mathbb{Z}^{n} \rightarrow \mathbb{N}^{d}$, from the graph vertices to $\mathbb{N}^{d}$, which is positive:

$\vec{k} \in \mathcal{D}_{m} \Rightarrow \rho(m, \vec{k}) \geq \overrightarrow{0}$ (component-wise) (Positivity)

and whose values stricly increase (according to $\rfloor_{d}$, the standard lexicographic order on integer vectors) at each edge $t=\left(m, m^{\prime}\right) \in \mathcal{T}:$

$$
Q_{m, m^{\prime}}\left(k, k^{\prime}\right) \Rightarrow \rho(m, \vec{k}) \prec_{d} \rho\left(m^{\prime}, \vec{k}^{\prime}\right) \quad \text { (Increasing) }
$$

It is said affine if it is affine in the second parameter (the variables $\vec{k}$ ). If $d>0$ the schedule is said to be multi-dimensional of dimension $d$.

In this paper, we restrict ourselves to the search of affine multidimensional schedules. In order to linearize both Positivity and Increasing constraints, we rely on the Farkas' Lemma, which we recall a statement below.

Lemma 3 (Farkas lemma, affine form). An affine form $\phi$ : $\mathbb{R}^{n} \rightarrow \mathbb{R}$ with $\phi(\vec{x})=\vec{c} \cdot \vec{x}+c_{0}$ is nonnegative everywhere in a non-empty polyhedron $\{\vec{x} \mid A \vec{x}+\vec{a} \geq \overrightarrow{0}\}$ iff:

$$
\exists \vec{\lambda} \in\left(\mathbb{R}^{+}\right)^{n}, \lambda_{0} \in \mathbb{R}^{+} \text {such that } \phi(\vec{x}) \equiv \vec{\lambda} \cdot(A \vec{x}+\vec{a})+\lambda_{0}
$$

The notation $\equiv$ is a formal equality, which means that we can deduce the equality of the affine coefficients.

$$
\exists \vec{\lambda} \in\left(\mathbb{R}^{+}\right)^{n}, \lambda_{0} \in \mathbb{R}^{+} \text {such that } \vec{c}=\vec{\lambda} \cdot A \text { and } c_{0}=\vec{\lambda} \cdot \vec{a}+\lambda_{0}
$$

Linearization of positivity constraints We now use this lemma on $\mathcal{D}_{m}=\left\{\vec{k} \mid D_{m} \vec{k}+\vec{d}_{m} \geq \overrightarrow{0}\right\}$ to "eliminates" the universally quantification on $\vec{k}$ from the constraints (Positivity):

$$
\begin{aligned}
& \exists \vec{\lambda}_{m} \in\left(\mathbb{R}^{+}\right)^{n}, \lambda_{m}^{0} \in \mathbb{R}^{+} \text {s.t. } \\
& \quad \rho(m, \vec{k}) \equiv \vec{\lambda}_{m} \cdot\left(D_{m} \vec{k}+\vec{d}_{m}\right)+\lambda_{m}^{0}
\end{aligned}
$$

which basically says that the schedule we are searching is expressed with the help of the constraints of the $\mathcal{D}_{m}$ domain. All the positivity constraints are thus linearized in the first half of Algorithm 5.

Linearization of edge $t=\left(m, m^{\prime}\right)$ increasing constraints First, we relax the increasing constraint (Increasing), for $d=1$, into:

$$
\left(\vec{k}, \vec{k}^{\prime}\right) \in Q_{t} \Rightarrow 0 \leq \rho\left(m^{\prime}, \vec{k}^{\prime}\right)-\rho(m, \vec{k}) \leq \epsilon_{t} \leq 1
$$

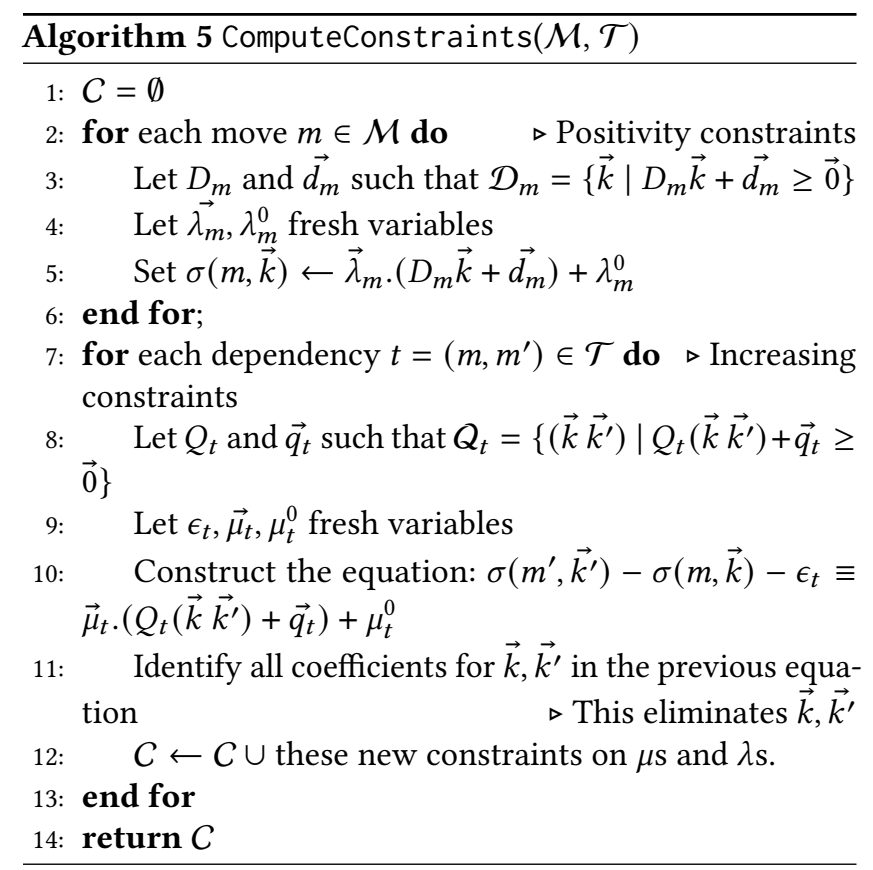

Applying Farkas' lemma to $Q_{t}=\left\{\left(\vec{k} \overrightarrow{k^{\prime}}\right) \mid Q_{t}\left(\vec{k} \vec{k}^{\prime}\right)+\vec{q}_{t} \geq \overrightarrow{0}\right\}$ :

$$
\begin{aligned}
& \exists \vec{\mu}_{t} \in\left(\mathbb{R}^{+}\right)^{n} \mu_{t}^{0} \in \mathbb{R}^{+} \text {s.t. } \\
& \quad \rho\left(m^{\prime}, \vec{k}^{\prime}\right)-\rho(m, \vec{k})-\epsilon_{t} \equiv \vec{\mu}_{t} \cdot\left(Q_{t}\left(\vec{k} \vec{k}^{\prime}\right)+\vec{q}_{t}\right)+\mu_{t}^{0}
\end{aligned}
$$

A substitution of (1) in (3) and an identification on each dimension of $\left(\vec{k} \vec{k}^{\prime}\right)$ leads to a set of linear inequalities that we denote by $C_{t}$. Algorithm 5 computes the union of all these constraints with the positivity constraints, and returns it. 